\title{
The study of nonlocal neural populations involving two neuron types and the effect of propofol
} Axel Hutt*1, Lutz Schimansky-Geier ${ }^{2}$ and Andre Longtin ${ }^{1}$

Address: ${ }^{1}$ Department of Physics, University of Ottawa, Ottawa, Ontario, Canada, K1N 6J7 and 2Institute of Physics, Humboldt University, Berlin, Germany

Email: Axel Hutt* - ahutt@uottawa.ca

* Corresponding author

from Sixteenth Annual Computational Neuroscience Meeting: CNS*2007

Toronto, Canada. 7-12 July 2007

Published: 6 July 2007

BMC Neuroscience 2007, 8(Suppl 2):SII doi:I0.1 I86/I47I-2202-8-S2-SII

(c) 2007 Hutt et al; licensee BioMed Central Ltd.

The work derives a neural population model, which considers excitatory and inhibitory synapses as well as excitatory and inhibitory neurons. Then the spatio-temporal dynamics of the neural population is studied subject to the increase of the inhibitory synaptic decay rate. This study is motivated by the effect of the anaesthetic propofol, which increases the inhibitory synaptic decay rate with increased blood concentration and may yield loss of consciousness. We find regimes of stationary multistability and stability criteria for the stationary states. It turns out that the increase and subsequent decrease of propofol yields saddle-node bifurcations in a hysteresis loop. 